Archives de sciences sociales des religions

151 | juillet-septembre 2010

Fondations des lieux de culte

\title{
Introduction: Procès de fondation
}

\section{Sossie Andézian}

\section{CpenEdition}

Journals

Édition électronique

URL : http://journals.openedition.org/assr/22306

DOI : 10.4000/assr.22306

ISSN : 1777-5825

Éditeur

Éditions de l'EHESS

Édition imprimée

Date de publication : 1 septembre 2010

Pagination : $9-23$

ISBN : 978-2-7132-2255-9

ISSN : 0335-5985

Référence électronique

Sossie Andézian, «Introduction: Procès de fondation », Archives de sciences sociales des religions [En ligne], 151 | juillet-septembre 2010, mis en ligne le 20 octobre 2010, consulté le 23 avril 2019. URL : http://journals.openedition.org/assr/22306 ; DOI : 10.4000/assr.22306 


\section{Sossie Andézian}

\section{Introduction : Procès de fondation}

En nous inspirant des travaux de Marcel Detienne (1990, 2009) sur la fondation des lieux, nous avons entrepris, à l'automne 2007, une réflexion sur les procès de fondation des lieux de culte dans le cadre d'un atelier du Centre d'études interdisciplinaires des faits religieux (CNRS/EHESS). Pas de contraintes de choix de religions ni d'aires géographiques et culturelles au départ. Un groupe d'une quinzaine de chercheurs, de post-doctorants et de doctorants s'est peu à peu constitué et a poursuivi ses activités pendant deux ans. Deux journées d'étude ont réuni l'ensemble des participants (les 11 et 12 juin 2009) pour présenter une première version des contributions dont onze sont publiées dans ce dossier. Une ligne directrice s'est dessinée dès les premières interventions et s'est confirmée par la suite : la fondation de lieux de culte comme moment d'émergence de nouveaux systèmes de sens et de création de nouveaux territoires, réels ou symboliques. Historiens, géographes, sociologues et anthropologues ont confronté données et analyses dans le domaine du judaïsme, du christianisme et de l'islam, dans des régions aussi variées que la France, les Antilles, les Balkans, le Proche-Orient et l'Asie du Sud. Les périodes historiques étudiées s'étalaient de la seconde moitié du XIX ${ }^{\mathrm{e}}$ siècle au début du XIX ${ }^{\mathrm{e}}$ siècle, avec des incursions plus loin dans le temps chaque fois que la mise en perspective des faits le nécessitait. Mur occidental du Temple de Jérusalem, églises, monastères, mosquées, mausolées, autant de formes différentes de monuments dans lesquels s'exerce le commerce avec le divin.

Précisons que les contributions se limitent aux religions du Livre, trois religions qui partagent cette caractéristique d'avoir refusé à leurs débuts de territorialiser la présence divine et posent donc le problème dans des termes communs et qui leur sont spécifiques. Chacune d'elle, en effet, voit ses édifices s'ériger et se multiplier à travers l'espace et le temps, paradoxe des trois religions, qui, tout en continuant de professer la transcendance, sont bien ancrées au sol ${ }^{1}$. Progressivement ces

1. Je renvoie le lecteur à l'excellent dossier de la Revue de l'histoire des religions «Lieux de culte, lieux saints dans le judaïsme, le christianisme et l'islam» (2005), coordonné par D. Iogna-Prat et G. Veinstein, dont la lecture peut être complétée par celle d'un ouvrage consacré spécifiquement à l'islam Lieux d'islam. Cultes et cultures de l'Afrique à Java (1996), dirigé par M. A. Amir-Moezzi. Sur l'histoire de la monumentalisation de l'Église chrétienne, on ne peut que recommander l'ouvrage de D. Iogna-Prat, La Maison Dieu (2006). 
constructions vont délimiter des territoires politiques et servir de support aux identités collectives, comme elles vont donner corps aux doctrines religieuses et exprimer les luttes et les conflits entre elles. Et leur transformation assez courante en lieux de mémoire leur confère un pouvoir symbolique de l'ordre de l'immanence, qui tend à confondre territoire sacré et entité politique.

Cette tendance s'est renforcée avec l'adoption, au XXe siècle, de la forme ÉtatNation suivant le modèle européen par la majorité des peuples, les lieux de culte devenant dans certains cas des enjeux dans les conflits de territoires entre nations antagonistes. Plus qu'une instrumentalisation du religieux par le politique, il s'agit de l'attestation de la force symbolique du religieux et de son usage comme facteur de transcendance et d'élévation au-dessus des contingences matérielles. Aussi, ce qui pouvait paraître incomparable au départ, en raison de spécificités nationales et religieuses, prend-il place dans un système d'équivalences, où des processus similaires d'articulation de deux éléments dichotomiques : le terrestre et le céleste, le matériel et l'immatériel, la forme et le fond, l'humain et le divin... sont observés, mais dont l'agencement s'opère chaque fois de façon singulière. Données géographiques, contextes historiques, facteurs politiques, économiques et culturels, idées religieuses interagissent diversement pour dessiner les contours des lieux du sacré et définir leurs caractéristiques et fonctions. C'est dire si l'on écarte la thèse chère à Mircea Eliade de la concentration du sacré en des lieux prédéterminés et sa transmission de façon quasi héréditaire ${ }^{2}$. Si des variables physiques entrent en jeu dans la détermination d'un site sacré (montagne, source, ravin, désert...), le caractère sacré n'est pas consubstantiel au site mais il résulte d'un ensemble d'opérations visant à le distinguer de son environnement. L'entrée en jeu des représentations religieuses, des croyances et des doctrines en précise l'identité et la vocation. Et c'est souvent aux moments de rupture (politique, économique ou religieuse) que celles-ci s'affirment, se reconfigurent ou laissent la place à de nouveaux systèmes de sens. La prise en compte de ces moments de rupture par l'ensemble des auteurs a rendu le travail de comparaison très pertinent. C'est également au cours de ces périodes que s'opèrent des passages du religieux au politique ou au culturel, processus que plusieurs articles s'attachent à décrire et analyser. Ce qui permet d'étendre et d'élargir les fonctions des lieux de culte en révélant la diversité des registres auxquels ils ressortissent et surtout leurs changements selon les contextes.

Malgré ces évolutions, un double processus était à l'œuvre dans chaque cas : construction physique et matérielle de monuments localisés et datés, et élaboration de traditions, de récits canoniques. Les enjeux de la fondation étaient recherchés dans le décalage entre faits historiques et récits mythiques ou légendaires, temps de l'histoire et temps des mythes ou des légendes.

2. Pour un examen critique des théories de la sacralité des sites, voir l'article de J.-P. Albert (2000). 
Les axes de réflexion s'organisaient peu à peu autour des questions suivantes :

Comment se constitue un lieu de culte ? Comment s'effectuent son individualisation et sa sacralisation ? Le lieu de culte territorialise-t-il plus que d'autres ? La fondation d'un lieu de culte constitue-t-elle un moment initial unique ? Quels types d'action des membres d'un groupe religieux (institutions et fidèles) mettentils en œuvre pour maintenir des liens avec ces lieux ?

Des éléments de réponses commençaient à surgir et des tendances se dessinaient :

La fondation de lieux de culte suit différentes étapes d'ancrage au sol ou d'enracinement de systèmes de sens, depuis l'appropriation et la délimitation d'un territoire dans un espace géographique donné, jusqu'à sa constitution en lieu consacré, en passant par la construction d'un édifice, l'invention d'une tradition lui conférant une légitimité, le choix d'une figure tutélaire et/ou d'une figure éponyme, l'élaboration d'une doctrine ou d'un ensemble rituel (ou le rattachement à une doctrine et à un ensemble rituel existants), la formation d'une communauté de fidèles, sa transformation en lieu de mémoire. Cette territorialisation contribue à la recomposition des espaces géographiques, politiques et culturels, des croyances et des identités des individus et des groupes sociaux qui les fréquentent. Aussi, la fondation de lieux de culte s'avère-t-elle être un objet pertinent d'analyse des rapports entre religion et territoire, religion et politique, religion et culture, appartenance religieuse et appartenance nationale et/ou ethnique.

Les travaux de Maurice Halbwachs et de Marcel Detienne nous ont guidés tout au long du parcours. Les thèses de Halbwachs sur la nécessité de localiser les représentations religieuses en des endroits précis pour les fixer dans la mémoire d'un groupe, développées pour expliquer l'attachement aux lieux saints chrétiens de Jérusalem, ont fait la preuve de leur transposabilité à d'autres religions et à d'autres contextes. Elles sont particulièrement pertinentes ici, où, comme on le verra, pour diffuser ou imposer de nouvelles idées religieuses, politiques ou culturelles, les hommes ont besoin de les matérialiser en les raccrochant à des lieux : "Il y a je ne sais quoi de mécanique dans la force qui retient les hommes autour d'un lieu consacré » (2008: 126).

Cependant, le lieu n'a pas la même configuration partout. Pour comparer les différentes situations, nous avons eu recours à la méthode proposée par Detienne «... comparer des comparables qui ne sont jamais immédiatement donnés et qui ne visent nullement à établir des typologies non plus qu'à dresser des morphologies » (2009: 11). L'idée de fondation n'est pas universelle, constatait Detienne ; elle est étrangère au Japon, en Inde et auprès des Indiens forestiers d'Amérique du Sud. Que comparait-il alors ? Ni des types de fondateur, ni des formes de territoire ou de maison ou de temple, mais des mécanismes de pensée qu'il repérait sous des entrées non thématiques «... que veut dire fonder, tracer un chemin, avoir des racines ou faire d'un lieu un non-lieu» (ibid. : 14). 
Nous ne comparons pas les manières de faire du territoire dans chaque religion comme il compare les manières de faire du territoire dans chacune des cultures étudiées. Sans tomber dans le relativisme, nous partons de quelques propositions générales sur des articulations possibles entre fondation de lieux de culte et systèmes de sens telles qu'elles se sont dégagées de l'ensemble des contributions. Elles ont différemment trait à la diffusion de nouvelles religions ou confessions, aux formes de territorialisation afférentes et aux usages politiques et culturels qui s’y rattachent. Si les auteurs partagent tous la caractéristique de travailler sur un même objet avec les mêmes concepts, les angles et les échelles d'observation privilégiés varient d'un article à l'autre. Mais presque tous sont soumis au jeu des échelles, avec déplacement de la focale pour mieux cerner l'objet dans sa complexité. Coutumiers de la pratique pluridisciplinaire, les auteurs se sont particulièrement attachés à se décentrer de leur discipline pour explorer d'autres facettes de leur objet. La contextualisation demeurait un souci permanent pour l'ensemble des contributeurs. Le travail comparatif a surtout consisté à isoler des spécificités historiques en partant d'une même action, par exemple «fonder un symbole religieux national ».

\section{Fondation et diffusion}

La fondation de lieux de culte est appréhendée comme l'expansion d'une nouvelle forme religieuse liée ou non à des conquêtes territoriales, à différentes échelles. Dans ce premier groupe d'articles, on suit l'aventure de la fondation de deux lieux de culte, une mosquée en Martinique (Liliane Kuczynski) et une église arménienne catholique à Jérusalem (Sossie Andézian). A priori rien ne semble rapprocher ces deux situations. Édification d'un lieu de culte musulman dans un pays à dominante chrétienne mais de constitution laïque, commencée dans les années soixante-dix dans un cas; d'un lieu de culte chrétien dans la Jérusalem ottomane à dominante musulmane et en cours de modernisation de la seconde moitié du XIX ${ }^{\mathrm{e}}$ siècle dans l'autre. La construction de l'édifice, non encore achevée pour la mosquée, durera près de cinquante ans pour l'église. Mais, fait intéressant, la délimitation et l'identification d'un territoire musulman à Fort-de-France et celles d'un territoire arménien catholique à Jérusalem se font très vite, dès le début de l'entreprise. Les auteurs se font particulièrement attentifs aux différentes étapes qui jalonnent le processus de construction, les obstacles qu'il rencontre, les conflits qu'il suscite. Et même si l'érection d'un monument demeure l'objectif ultime, l'accent est mis sur la nécessité d'implantation d'une nouvelle religion (islam) ou d'un nouveau rite (arménien catholique). Dans l'un et l'autre cas le nombre de fidèles n'est pas important (environ cinq cents musulmans en Martinique et quatre familles arméniennes catholiques à Jérusalem). Le lieu précède la communauté et devrait servir de pôle d'attraction pour ses membres et les futurs convertis. Pas de développement ni de diffusion sans un territoire spécifique. 
L'histoire du travail de construction révèle la complexité du champ religieux et la compétition entre forces concurrentes au sein d'une même religion. Si les deux projets se heurtent aux résistances des autorités publiques à implanter des lieux de culte de religions minoritaires dans un premier temps, les difficultés viendront surtout de l' "intérieur ", du monde musulman et du monde chrétien (puis catholique). Et le projet de construire un lieu de culte à l'intention des fidèles cède le pas à la mainmise de courants religieux se réclamant de l'orthodoxie et de l'orthopraxie, ou à des tentatives de centralisation pour pallier la fragmentation. Il se transforme clairement en projet d'imposition d'une religion normative qui cherche à gommer les spécificités ethniques et nationales, mais aussi les particularités rituelles au sein d'une même famille religieuse. Du coup, il déborde du cadre local pour s'intégrer dans le cadre plus global de la diffusion d'une religion donnée dans un nouveau contexte national de diversité religieuse. C'est le problème que connaît aujourd'hui l'implantation de l'islam en Occident, les autorités publiques ayant du mal à gérer la double contrainte du respect de la liberté religieuse de leurs ressortissants et de l'affiliation de ces derniers à une religion à visée universaliste. La fondation d'églises catholiques de rite oriental avait connu le même dilemme, surtout au XIX ${ }^{\mathrm{e}}$ siècle : comment créer de nouvelles Églises nationales au sein d'une Église universelle, sans perdre sa langue et ses traditions tout en rompant avec les Églises-mères porteuses de cette mémoire des origines. Comme on le verra ici, les rapports de l'Église arménienne catholique avec l'Église arménienne apostolique d'un côté, avec l'Église catholique universelle, de l'autre, ne seront pas de tout repos. Des conflits éclateront au sein même de l'Église arménienne catholique entre partisans et opposants de l'option centralisatrice, aussi bien au sein du clergé que parmi les laïcs.

Dans l'un comme dans l'autre cas, la construction d'un lieu de culte met en scène la fragmentation des communautés religieuses respectives. Alors qu'habituellement un lieu de culte est perçu comme un centre fédérateur, symbole d'unité, ici il se révèle comme analyseur des contradictions inhérentes à la constitution de lieux représentatifs de groupes humains liés par l'appartenance. La question de l'autochtonie est au cœur de ce questionnement et suscite le débat dans les deux exemples. Il est significatif que le choix de la figure du fondateur se fixe sur des personnages locaux, qui, tout en œuvrant activement à la diffusion de religions universalistes, s'attachent à la défense des identités locales, et plutôt que l'absorption dans l'islam ou le catholicisme universels, s'emploient à leur vernacularisation. Il ne s'agit pas d'une posture autonomiste mais d'une stratégie d'intégration par le bas au sein de religions universelles.

Ce débat sur l'autochtonie se reflète dans l'architecture, qui mélange caractéristiques spécifiques aux mosquées (minaret) et aux églises catholiques (plan intérieur en croix latine) et style architectural ethnique et/ou national (duplication de la mosquée de la ville palestinienne d'al-Bireh et autel surmonté du clocher octogonal des églises arméniennes). 
Cette appropriation locale d'une religion universelle est très bien illustrée dans la contribution de Willy Jansen et Catrien Notermans. Ici pas de lieu de culte du rite qu'on souhaite développer (le rite latin de langue arabe), mais un projet éducatif, celui des filles palestiniennes par des religieuses du pays. Or celles-ci n'existent pas. Il faut les créer. La fondation d'une congrégation religieuse féminine palestinienne va se faire avec l'aide de la Vierge, qui apparaît à une religieuse palestinienne de la congrégation française de Saint-Joseph de l'Apparition, Sœur Marie-Alphonsine. L'événement a lieu en 1874 -1875, à la suite des apparitions mariales attestataires en France (Lourdes, La Salette, Pontmain). C'est aussi l'époque de l'arrivée massive des congrégations religieuses en Palestine, d'abord masculines puis féminines. On est, tout simplement, en pleine tentative d'expansion du catholicisme en Terre sainte pour contrebalancer la présence orthodoxe et protestante. Le Consul de France, protecteur des catholiques de l'Empire ottoman et le patriarche latin ont réussi à racheter ou à se faire offrir des terrains Via Dolorosa, sites de vestiges d'églises byzantines ou croisées, pour reconstituer le chemin de la Passion. Dans ce cadre, les Arméniens catholiques ont pu acquérir les $\mathrm{III}^{\mathrm{e}}$ et $\mathrm{IV}^{\mathrm{e}}$ stations. La naissance de la congrégation des sœurs du Rosaire, nom donné en référence à l'image de la Vierge apparue un chapelet à la main, prend place dans le mouvement d'indigénisation du clergé impulsé par le Vatican. C’est que les conversions au rite latin de la population chrétienne autochtone, majoritairement orthodoxe ou de rite arménien, ne sont pas aussi nombreuses. Pour la hiérarchie de l'Église catholique, il ne s'agit pas simplement de gagner de nouveaux fidèles mais de " ramener dans son giron les schismatiques » et de réunir les chrétiens.

Contrairement à l'exemple de l'Église arménienne catholique, qui fonde sa présence en Terre sainte autour d'un centre de pèlerinage sur les traces de la Vierge, l'Église latine de langue arabe, en pleine expansion, mise son existence sur la formation catholique des filles palestiniennes, futures mères et éducatrices. La congrégation du Rosaire, dont les écoles se sont répandues dans plusieurs pays du Moyen-Orient, est à ce jour une des meilleures institutions éducatives destinées aux filles. Ces établissements sont fréquentés aussi bien par des chrétiennes de tous rites que par des musulmanes. Et si le projet de conversion des élèves au catholicisme n'est plus d'actualité, reste le souci de sauvegarder l'identité des chrétiens arabes devenus très minoritaires. La béatification, à Nazareth, le 22 novembre 2009, de la fondatrice de la congrégation, une enfant du pays, que la hiérarchie de l'Église catholique a interprétée comme un signe divin à l'intention des chrétiens de Terre sainte vivant dans des conditions difficiles et menacés d'extinction, est supposée leur redonner de l'espoir et les encourager à rester dans ce berceau du christianisme, non plus pour diffuser le catholicisme oriental comme au XIX ${ }^{\mathrm{e}}$ siècle, mais pour témoigner de leur foi malgré leur petit nombre.

Si les deux premiers articles se focalisent sur l'analyse de la fondation d'un lieu de culte comme l'expression de l'inscription d'une religion nouvelle dans le 
paysage et comme medium pour sa diffusion, le troisième montre qu'une religion peut s'inscrire et se diffuser par d'autres canaux que des monuments, en l'occurrence un système d'enseignement.

\section{Territoires et identités}

Les quatre articles suivants appréhendent un autre aspect du processus de fondation de lieux de culte : la participation à la fabrique des identités collectives politico-religieuses dans des sociétés du Moyen-Orient contemporain, en Jordanie, Syrie, Liban (Norig Neveu, Anna Poujeau, Emma Aubin-Boltanski), mais aussi dans les Balkans, dans l'Albanie de l'entre-deux-guerres (Nathalie Clayer). Les religions en jeu sont, là encore, l'islam et le christianisme oriental. Mais, comme on le verra, les modalités d'articulation du religieux et du politique ne diffèrent pas trop d'une situation à l'autre. Le recours au passé, historique ou mythique, se fait selon le schéma classique des nations modernes, par l'invention d'une tradition à travers un tri sélectif d'éléments considérés comme significatifs.

Le phénomène est envisagé à l'échelle de la nation (création d'une identité nationale musulmane en Albanie ; invention de la Terre sainte musulmane en Jordanie) ou à l'échelle d'un groupe de population appartenant à une religion minoritaire dans le pays (les chrétiens de Syrie) ou encore à l'échelle d'un village (les maronites de Béchouate dans une région à dominante musulmane chiite), ou plus exactement d'une famille du village. Les monuments, construits, reconstruits ou rénovés, sont la marque de cette affirmation identitaire confessionnelle dans l'espace public. Leur légitimation par leur inscription dans l'histoire religieuse longue du pays, de la région ou du village, les enracine dans la terre des ancêtres, conçue comme intemporelle.

L'intérêt de ces contributions est de rendre compte des logiques de constitution d'identités collectives à un moment précis de l'histoire de chacun des pays étudiés, caractérisé par la nécessité de construire la nation (Albanie) ou de la consolider lors de crises internes entraînant l'émergence d'identités singulières, politiques ou religieuses (Jordanie, Syrie, Liban). Les édifices apparaissent comme l'expression par excellence du rapport au territoire et en assurent la cohésion. La comparaison entre les quatre situations éclaire d'un nouveau jour les usages politiques du religieux dont on pensait avoir épuisé l'analyse. La focalisation sur les processus de création de symboles nationaux de nature religieuse tels que les lieux de culte, tout en confirmant la force de mobilisation du religieux, fournit la preuve du caractère non essentiel de la sacralité de ces lieux. Elle met en évidence le rôle des représentations dans la démarche de sacralisation des lieux de culte et des territoires dont ils assurent le maillage. La notion de frontière est fondamentale ici. Il s'agit en effet de tracer des limites : avec les pays voisins, pour s'en distinguer nettement et surtout pour afficher une identité singulière (l'Albanie par rapport à la Grèce, la Jordanie par rapport à Israël); avec les co-nationaux dont on se 
différencie par l'appartenance religieuse (les chrétiens de Syrie par rapport aux musulmans du pays); avec les co-nationaux religieusement autres mais avec lesquels on partage une région spécifique (les maronites du village de Dayr alAhmar de la Bekaa au Liban par rapport à leurs voisins chiites).

Si les mêmes mécanismes président à la mise en relation de la fondation de lieux de culte avec des représentations du passé et du patrimoine dans les quatre cas, la configuration qui en résulte est particulière chaque fois, des spécificités historiques et des contextes politiques différentiels imprimant leur propre marque.

Nathalie Clayer analyse les enjeux de la construction de mosquées dans l'Albanie de l'entre-deux-guerres, jeune État-nation déclaré laïque, où la religion est soumise à son contrôle. L'association entre l'appartenance à l'islam de la majorité de la population $(70 \%)$ ne semble pas aller de soi dans ce pays inscrit dans l'Europe et entouré de pays chrétiens. C'est ce paradoxe que l'auteure tente d'éclairer. Elle fait apparaître les mosquées comme des lieux de débat entre religieux et laïcs sur la place de la religion en général, et de l'islam en particulier, dans la définition de l'identité albanaise. On perçoit de la sorte les tensions entre autorités politiques et autorités religieuses pour déterminer la nature de l'islam albanais, ainsi que les divergences entre représentants religieux conservateurs et réformistes. L'examen du processus d'édification de mosquées dans trois régions, le Nord, le Centre et le Sud du pays, met en évidence des enjeux différents (intercommunautaires, nationaux ou internationaux), mais étroitement liés à des questions territoriales. Soutenues, sinon toujours financièrement, mais du moins symboliquement par les pouvoirs politique et religieux, ces mosquées, matérialisation de l'image d'un islam réformé et moderne, sont supposées assurer la légitimité de l'État albanais en Europe.

Dans l'exemple jordanien, le maillage du territoire, avec des mosquées, des tombes des Compagnons du Prophète et des tombes de saints, intervient plus tard, dans le contexte d'une recomposition politique régionale et de la compétition pour le contrôle de la Terre sainte entre Israël, l'Autorité palestinienne et la Jordanie ${ }^{3}$. Sur le plan intérieur, cette initiative vient affirmer l'identité islamique du pays, non seulement par l'adoption de l'islam comme religion d'État, mais par le rattachement de la famille royale à celle du Prophète. Les raisons économiques n'en sont pas négligeables puisqu'il s'agit de développer le tourisme religieux au niveau régional et international, aussi bien vers des sites chrétiens que vers des sites musulmans. La Jordanie convoite ainsi la première place parmi les pays arabes de la région en tant que porte-parole de l'islam et plus récemment en tant que promoteur du dialogue islamo-chrétien, rôle plus naturellement dévolu au Liban de par le caractère interconfessionnel de son système politique, que leur

3. La fondation de tombes des Compagnons du Prophète ayant combattu pour l'expansion de l'islam constitue un processus de légitimation très courant dans l'emprise de cette religion sur un territoire (Veinstein, 2005). 
dispute également la Syrie jusqu'à un certain point. Les monuments sont ici polysémiques : ils disent la nation (islamique, de descendance prophétique), en définissent les limites (principalement le long de la frontière israélo-jordanienne); leur style architectural s'inspire de monuments de pays musulmans tout en se voulant moderne.

On retrouve les mêmes logiques qu'en Albanie, mais ici le mouvement est d'une plus grande ampleur et les référents islamiques sont plus nets. Centrée sur la politique de la famille régnante et des institutions officielles, l'étude laisse toutefois entrevoir les débats qu'une telle action suscite, avec les islamistes d'une part, avec les habitués des lieux de culte transformés et dépossédés de leurs fonctions traditionnelles, d'autre part.

Sur les chrétiens de Syrie, Anna Poujeau adopte une autre perspective en proposant une analyse du point de vue des sujets, qu'elle situe par la suite dans le cadre de la politique de l'État envers les minorités religieuses du pays. Elle traite du rôle des monastères dans l'inscription des chrétiens dans le paysage national. On voit comment le renouveau monastique, engagé dans le pays depuis plusieurs années, provoque l'émergence d'un discours nationaliste chrétien faisant des monastères les éléments fondateurs du territoire syrien et de son identité. Défendant l'idée de l'antériorité de leur présence dans le pays par rapport aux musulmans, majoritaires depuis plusieurs siècles, les chrétiens se définissent comme les véritables autochtones.

La réflexion de l'auteure sur les représentations des monastères dans l'imaginaire chrétien révèle leur force symbolique pour des populations numériquement minoritaires et exclues du jeu politique en tant que collectivité. Comme si la visibilité accrue de ces lieux de culte aujourd'hui avec leurs signes ostentatoires (croix géantes illuminées) ${ }^{4}$ ne suffisait pas à asseoir leur légitimité dans le pays, les chrétiens se réinventent un passé d'autochtones fixés sur un territoire formé de monastères qui se fondent dans le paysage. Ensevelis ou à l'état de ruine, ces édifices constituent le mythe d'origine de la Syrie telle qu'ils se la représentent. Ainsi, on peut dire que ce sont les monastères, constructions réelles ou imaginaires, qui ancrent les chrétiens dans le territoire syrien actuel et futur. Cette thèse est confirmée par l'usage que ces derniers font de ces établissements religieux aujourd'hui, lieux de pratique religieuse préférés aux paroisses, où les fidèles peuvent confier leurs problèmes à la Vierge et aux saints, lieux de promenade,

4. À noter le développement, dans le monde entier, aujourd'hui, de constructions de nouveaux lieux de culte plus ou moins imposants dans les centres urbains, qui tendent à transformer la dynamique des villes. Ces monuments deviennent les marqueurs de la présence de certains groupes sociaux qui s'affirment ainsi par des signes religieux ostentatoires ( $c f$. : Les Annales de la recherche urbaine, "Urbanité et liens religieux ", P. Lassave, A. Querrien, (dirs.), 96, 2004 ; Topographie du sacré. L'emprise religieuse sur l'espace, A. Morelli, (dir.), 2008 ; Revue des mondes musulmans et de la Méditerranée (REMMM), "Les mosquées. Espaces, institutions et pratiques », F. Adelkhah, A. Moussaoui, (dirs.), 125, 2009). 
de convivialité et d'expérience de l'appartenance communautaire. Leur valorisation par les prélats, notamment par le patriarche grec orthodoxe, chef d'une communauté qui fut le chantre du nationalisme arabe à ses débuts, confère davantage de valeur à ces institutions religieuses devenues intemporelles. L'historiographie nationale, qui tend à gommer la période islamique afin de réduire les différences religieuses et de promouvoir l'identité arabe du pays, les légitime indirectement.

Emma Aubin-Boltanski présente un autre exemple d'usage de lieux de culte par des chrétiens, maronites, pour s'inscrire dans une région, la Bekaa, dominée par une majorité chiite. À partir de l'étude du centre de pèlerinage de NotreDame de Béchouate, elle s'attache à analyser les modalités d'usage du mythe fondateur du lieu de culte et ses relations avec le mythe fondateur du village, étroitement lié à une épopée familiale, celle des Kayrouz. La consignation du mythe fondateur dans plusieurs textes à des époques différentes lui confère plus de légitimité aux yeux des habitants, qui le mobilisent en cas de nécessité. Les variations des données d'une version à l'autre reflètent le caractère dynamique du processus de fondation de lieux de culte qui tient compte de l'évolution des contextes sociaux et politiques.

Mais ici, l'affirmation de la présence chrétienne n'est pas à usage interne comme chez les chrétiens de Syrie. Elle fait face à l'autre, le musulman, qui de plus est sollicité comme garant de son existence. La participation, plus récemment, du lieu au débat sur le dialogue interreligieux au Liban le transforme en symbole national, voire international, tant ce débat s'étend en Orient et en Occident depuis le 11 septembre 2001. Cette dynamique de constitution de Notre-Dame de Béchouate en symbole de la coexistence islamo-chrétienne en vue de favoriser l'union nationale en est encore à ses débuts. Ce sanctuaire pourrait-il devenir un des hauts lieux du dialogue interreligieux ? L'auteure souligne le rôle de la Vierge en tant que " médiatrice " et " régulatrice » dans les relations interreligieuses, et le maire du village attribue à la Vierge la fonction de chef religieux et de chef politique. Toute la problématique des habitants du village se résume dans ce sanctuaire, lequel, comme les monastères chrétiens de Syrie, est imaginé comme étant constitutif de la géographie à la fois physique, mentale et spirituelle. Il est ainsi au fondement de leur existence ${ }^{5}$.

5. Les rapports entre religion et territoire ont été abondamment étudiés par les anthropologues et les historiens, qui se sont surtout interrogés sur le rôle des religions dans la formation des sociétés humaines. On citera pour exemple l'ouvrage de Maurice Godelier, au titre éloquent "Au fondement des sociétés humaines " (2007), qui démontre que ce sont les rapports politicoreligieux, et non les rapports de parenté, ni les rapports économiques, qui forment les sociétés. 


\section{Idéologies " séculières " et lieux de culte}

Dans l'histoire des lieux de culte, apparaissent des moments où des laïcs, intellectuels ou hommes politiques, se les approprient, élaborent et diffusent leur propre idéologie et leur propre conception du religieux. C'est à une telle réflexion que nous convient les contributions ci-après. Simone Ricca analyse les transformations du Mur des Lamentations, ou Mur occidental, dans la vieille ville de Jérusalem du point de vue architectural et urbain visant à en faire l'élément clé de la « capitale éternelle » du pays; de leur côté, Michel Boivin et Rémy Delage proposent une lecture de la naissance d'un culte soufi « sécularisé » dans le mausolée de Benazir Bhutto, ancien premier ministre du Pakistan, morte assassinée et déclarée martyre par ses partisans ; Frédéric Gugelot s'attache à mettre en lumière les processus d'appropriation du phénomène de Lourdes par des écrivains au tournant $\mathrm{du} \mathrm{XX}^{\mathrm{e}}$ siècle et rend compte du débat d'idées en cours dans la société ; Brigitte Bleuzen, enfin, nous livre les résultats d'une enquête en cours sur les mutations du pèlerinage médiéval breton aux sept saints (Tro-Breiz), ainsi que le projet de construction de la Vallée des Mille Saints.

Tous ces lieux se trouvent investis de nouvelles significations et de nouvelles fonctions, ce qui atteste du caractère dynamique de leur formation, et dont on ne peut saisir toute la complexité que dans la diachronie. Ces contributions présentent l'intérêt de souligner que la manipulation du sacré n'est pas le monopole des religieux : elle peut être tout autant le fait de laïcs, hommes de pouvoir, penseurs ou simples citoyens. Tout en s'appuyant sur la mémoire établie des lieux, ces derniers entreprennent leur transformation physique (Mur, Tro-Breiz) et symbolique (dans les quatre cas). Toutefois, il ne s'agit pas d'un simple phénomène de sécularisation. Le sacré est fortement mobilisé, mais il est réinterprété, déplacé, transféré. Les frontières entre religion et politique, religion et culture sont brouillées.

Simone Ricca suit l'évolution du statut et des significations du Mur occidental, qui connaît un changement radical en 1967 avec l'occupation de Jérusalem-Est et des territoires palestiniens par Israël. On assiste à l'invention d'un lieu saint à des fins politiques, l'État cherchant à l'ériger en symbole de l'union nationale. Le quartier musulman ou quartier des Maghrébins est détruit pour étendre l'espace sacré. Ce site, que les musulmans revendiquent également comme un lieu saint, celui de l'Ascension du Prophète, devient un espace sacré juif exclusivement. Francis Schmidt (1994) avait montré le rôle joué par le Temple dans la cohésion sociale du peuple juif. Non seulement comme édifice et comme lieu de pèlerinage avec ses infrastructures, mais comme pensée avec ses catégories du pur et de l'impur, du sacré et du profane qui touchaient tout le pays et tous les espaces de vie. Après sa destruction, en 70, lorsque traditions et rituels disparaissent, demeure le Temple comme lieu de rassemblement de l'ensemble du peuple juif. Mais surtout la pensée du Temple. Comment cette pensée se concrétise-t-elle après 1967 ? 
Si de plus en plus de Juifs d'Israël et de la diaspora viennent prier dans cette synagogue à ciel ouvert, l'objectif de l'État est politique : faire de ce lieu le symbole du sionisme. D'où la création, dans le périmètre, d'une place pour les manifestations civiques et militaires, qui, avec les rituels religieux, devraient contribuer à symboliser la nation en unissant toutes ses composantes et en faire une figure de rhétorique du territoire national ${ }^{6}$. Or, progressivement, le lieu échappe aux politiques, il est délaissé par les laïcs et approprié par des organisations religieuses plus ou moins radicales. Les femmes sont exclues de certaines parties, les Israéliens non juifs n'y sont pas admis, sauf en touristes et sous certaines conditions. Aussi, au lieu de constituer un symbole d'union nationale, le Mur devient le révélateur des failles au sein de la nation.

Cette même dynamique d'utilisation des lieux saints dans le jeu politique est mise en évidence dans l'exemple présenté par Michel Boivin et Rémy Delage. Là encore, ce sont des référents religieux - le système symbolique du soufisme et la notion chiite de martyre - qui sont mobilisés par la famille Bhutto pour asseoir son pouvoir politique à l'échelle de la nation. Le mausolée familial, où sont enterrés plusieurs membres de cette lignée d'hommes politiques morts assassinés (martyrs), associe les deux registres religieux et politique : à l'intérieur s'exerce la piété soufie et à l'extérieur le pouvoir politique. L'architecture et l'iconographie appartiennent au registre du soufisme. Mais la tentative de transformation du mausolée en lieu de commémoration nationale échoue : celui-ci devient un lieu de pèlerinage régional seulement. Comme le Mur occidental, il symbolise moins l'union nationale que ses divisions. Par conséquent, il est loin de constituer une figure de rhétorique du territoire national.

Ces deux exemples confirment la force de mobilisation des lieux de culte par des instances politiques en quête de légitimité. Ils révèlent en même temps la fragilité de l'association des registres politique et religieux, qui, pour se stabiliser, a besoin d'être légitimée par la base. Or, dans un cas comme dans l'autre, les significations du lieu imposées par les autorités politiques sont en décalage par rapport aux représentations religieuses des fidèles qui se fondent sur des traditions établies et sur leur mémoire. Son appropriation exclusive par une seule composante de la société ne peut qu'en faire un lieu fermé, inaccessible à ceux " qui n'en sont pas » et par conséquent lieu d'affrontement entre diverses conceptions du religieux et du politique.

Cette absence de consensus sur la signification des lieux de culte constitue l'objet de l'article de Frédéric Gugelot qui, en s'appuyant sur l'abondante production littéraire sur Lourdes, démontre que ce lieu s'est construit autant en pierre qu'en papier. Il souligne le pouvoir de ces œuvres dans le maintien d'une atmosphère de mystère, garante de la pérennité du lieu. L'auteur analyse en particulier deux

6. Expression empruntée à B. Debarbieux (1995) qui fait référence à des lieux symboliques permettant d'évoquer le territoire. 
œuvres, Lourdes de Zola et Les foules de Lourdes de Huysmans. Respectivement fondateur et adepte du naturalisme, tous deux mènent une enquête sur le terrain afin de saisir le phénomène dans sa globalité de manière objective. Zola le sceptique et Huysmans le croyant éprouvent la même fascination pour la foi des foules et font le constat de la primauté de l'affect sur la raison. Les deux auteurs associent le succès du lieu à la charge émotionnelle qui s'en dégage.

À travers la confrontation des positions divergentes des deux écrivains, Gugelot fait l'état des lieux des courants de pensée au tournant $\mathrm{du} \mathrm{XX}^{\mathrm{e}}$ siècle ainsi que de la place de la religion dans la société. Si l'un et l'autre admettent que Lourdes est une réponse au besoin de croire des foules, Zola prône la transmutation du sentiment religieux en faveur d'une religion vidée de tout dogme, tandis que Huysmans plaide pour une nouvelle conception de la religion, beaucoup plus intérieure. L'interprétation culturelle du phénomène Lourdes dans sa diversité vient s'ajouter à l'expérience des foules pour complexifier davantage le lieu et ses attributs et lui conférer un caractère dynamique ouvert au changement. Si elle ne réussit pas à en supplanter la dimension religieuse, elle a le mérite d'élargir les cadres de l'expérience, qui ne se limitent plus à un système de croyances, de dogmes et un nombre fini de pratiques. Tout en conservant ses caractéristiques physiques, le lieu de pèlerinage devient le réceptacle d'une multiplicité de représentations et un terrain favorable à l'invention de nouveaux types de rapports avec le surnaturel.

Cette plasticité des lieux de culte trouve toute son expression dans l'article de Brigitte Bleuzen. Nouvelle version du pèlerinage aux sept saints fondateurs de la Bretagne, démarrée en 1994 à l'initiative de laïcs et avec le soutien du Vatican, le Tro-Breiz (le tour de la Bretagne) oscille entre univers religieux et univers culturel. La frontière reste floue entre marche et pèlerinage, entre croyants et non-croyants. Il en est de même du projet d'érection de mille statues en hommage aux saints, personnages de légendes protecteurs du pays. Mais pas de doctrine, ni de dogme, ni de pratiques codifiées. L'enquête illustre la mutation du rapport au religieux : glissement de l'appartenance à la paroisse au lien social basé sur l'identité bretonne valorisant l'amour $\mathrm{du}$ " pays ». Ce rapport au pays qui entretient une part de mystère et célèbre la mémoire des ancêtres est de nature religieuse, soutient l'auteure, non seulement parce qu'il s'agit de saints mais parce qu'il comporte une dimension d'expiation du mal fait à la terre par les hommes. C'est une religion redéfinie par l'homme, en fonction de ses propres attentes, et non imposée par une Église ou des instances organisées, qui se confond avec la nature et la culture pour servir un seul objectif : la re-sacralisation de la Terre-Mère bretonne meurtrie.

Zola et Huysmans avaient appelé de leurs vœux, chacun à sa manière, cette reprise en mains de leur destin par les hommes pour conférer de nouveaux sens aux mystères de la nature. Il est intéressant de noter qu'un siècle plus tard, les problématiques abordées par ces deux écrivains sont toujours d'actualité et témoignent du besoin qu'ont les hommes de changer les cadres institués de l'expérience religieuse. 
L'histoire des lieux de culte est jalonnée de moments de fondation et de refondation. Leur vocation change au gré des contextes historiques et politiques. Manifestations concrètes d'actions d'expansion religieuse, ils cristallisent tensions internes et externes et traduisent la dynamique d'émergence de nouvelles formes religieuses. Leurs usages politiques les situent au fondement de territoires nationaux ou locaux et les transforment en mythes d'origine de groupements humains. Traversés par les débats en cours sur la place de la religion dans la société, ils reflètent les mutations qui affectent les représentations du sacré. On voit notamment que le rapport au surnaturel n'est plus le monopole des religions instituées et de leurs spécialistes comme ce fut massivement le cas jusqu'à notre époque dans les religions du Livre. La religion n'est pas reléguée, loin s'en faut, mais elle s'insère dans un ensemble de systèmes de sens ressortissant à divers domaines.

Les lieux de culte se révèlent comme des espaces de régulation des controverses, polémiques et conflits que ces situations de changement suscitent. N'est-ce pas leur fonction de réceptacle des mémoires des générations successives qui leur confère ce rôle de médiateurs, entre différents systèmes de sens ; entre religion et société ; entre passé, présent et futur et les inscrit dans la durée ?

Sossie ANDÉZIAN

\section{Bibliographie}

Adelkhah F., Moussaoui A., (dirs.), 2009, «Les mosquées. Espaces, institutions et pratiques ", numéro spécial de la Revue des mondes musulmans et de la Méditerranée (REMMM), 125.

AlbERT Jean-Pierre, 2000, "Des lieux où souffle l'esprit », Archives de sciences sociales des religions, 111, pp. 111-123.

AmIr-Moezzi Mohammad Ali, (dir.), 1996, Lieux d'islam. Cultes et cultures de l'Afrique à Java, Paris, Autrement, coll. "Mémoires/Histoire».

DEBARBIEUX Bernard, 1995, "Le lieu, le territoire et trois figures de rhétorique ", L'espace géographique, 2, pp. 97-112.

DetienNe Marcel, (dir.), 1990, Tracés de fondation, Louvain-Paris, Peeters, coll. « Bibliothèque EPHE Sciences religieuses ", CXIII.

-, [2000] 2009, Comparer l'incomparable. Oser expérimenter et construire, Paris, Seuil, coll. « Points ».

Dierkens A., Morelli A., (dirs.), Topographie du sacré. L'emprise religieuse sur l'espace, 2008, Bruxelles, Éditions de l'Université de Bruxelles, coll. «Problèmes d'histoire des religions ", XVIII.

GODELIER Maurice, 2007, Au fondement des sociétés humaines. Ce que nous apprend l'anthropologie, Paris, Albin Michel, coll. "Bibliothèque Idées ».

HALBWACHS Maurice, 2008, La topographie légendaire des Évangiles en Terre sainte. Étude de mémoire collective, (Édition préparée par Marie Jaisson), Paris, Presses Universitaires de France (1 ${ }^{\text {ère }}$ éd. 1941). 
Iogna-Prat Dominique, 2006, La Maison-Dieu : Une histoire monumentale de l'Église au Moyen Âge (v. 800-v. 1200), Paris, Seuil.

Iogna-Prat Dominique, Veinstein Gilles, (dirs.), 2005, "Lieux de culte, lieux saints dans le judaïsme, le christianisme et l'islam ", numéro spécial de la Revue de l'histoire des religions, 222-4.

LASSAVE Pierre, QUERRIEN Anne, (dirs.), 2004, «Urbanité et liens religieux », numéro spécial de Les Annales de la recherche urbaine, 96.

SCHMIDT Francis, 1994, La pensée du Temple. De Jérusalem à Qoumrân. Identité et lien social dans le judaïsme ancien, Paris, Seuil, coll. "La Librairie du XX $\mathrm{X}^{\mathrm{e}}$ siècle ».

Veinstein Gilles, 2005, "Le rôle des tombes sacrées dans la conquête musulmane ", in Iogna-Prat D., Veinstein G., (dirs.), « Lieux de culte, lieux saints dans le judaïsme, le christianisme et l'islam », numéro spécial de la Revue de l'histoire des religions, 222-4, pp. 509-528. 